\title{
Structure and significance of cruciate flagellar root systems in green algae: Female gametes of Bryopsis lyngbyei (Bryopsidales)
}

\author{
M. Melkonian \\ Botanisches Institut, Westfälische Wilhelms-Universität; \\ Schloßgarten 3, D-4400 Münster, Federal Republic of Germany
}

\begin{abstract}
The ultrastructure of the flagellar apparatus in the biflagellate female gametes of the green alga Bryopsis lyngbyei has been studied in detail. In the flagellum and basal body, microtubule septations occur in some of the B-tubules. The transition region of the flagellum is extremely long $(260-290 \mathrm{~nm})$, exhibits a stellate pattern in cross section but lacks the transverse diaphragm. The two basal bodies form an angle of $180^{\circ}$ and overlap at their proximal ends. They are connected by a compound non-striated capping plate. Terminal caps associated with the capping plate partially close the proximal end of each basal body. A cruciate flagellar root system with three different types of microtubular roots is present, i. e. the flagellar apparatus does not show $180^{\circ}$ rotational symmetry. One root type contains 2 microtubules which are connected to an elaborate cylindrical structure, presumably a mating structure. The opposite root exhibits 3 microtubules over its entire length and is not associated with a cylindrical structure. In their proximal parts both roots are linked to an underlying crescent body. The other two microtubular roots are probably identical and consist of 4 (or 5) microtubules which show configurational changes. These two identical roots insert into the capping plate and link to the inner side (i. e. the side adjacent to the other basal body) of each basal body, whereas the other two roots attach to the outer sides of each basal body. System I striated fibres are probably associated with each of the four roots, while system II fibres have not been observed. The flagellar apparatus of female gametes of $B$. lyngbyei shows many unique features but in some aspects resembles that of ulvalean algae. Functional and phylogenetic aspects of cruciate flagellar root systems in green algae are discussed.
\end{abstract}

\section{INTRODUCTION}

In the past few years the detailed uitrastructure of the flagellar apparatus has been increasingly used as a phylogenetic indicator in the green algae (summaries by Moestrup, 1978; Melkonian, 1980a). These studies have complemented and extended earlier investigations on mitotic and cytokinetic mechanisms in green algae (summarized by Pickett-Heaps, 1975; Stewart \& Mattox, 1975). The postulation of a third class of green algae (besides the Chlorophyceae and Charophyceae sensu Stewart \& Mattox), the Ulvaphyceae sensu Stewart \& Mattox, was mainly based on cytokinetic properties and presence of scales on motile cells of some species (Stewart \& Mattox, 1978). In the past two years it has also been shown that the flagellar apparatus of the Ulvaphyceae sensu 
Stewart \& Mattox contains some special structures confined to motile cells of this green algal group (Melkonian, 1979; Sluiman et al., 1980; Melkonian, 1980b; Hoops et al., 1981). One result of these studies has been the finding that fibrous structures associated with basal bodies exhibit a great diversity and are potentially more useful as phylogenetic markers compared to the rather uniform microtubular flagellar root system (see also discussion in Melkonian, 1980a). The uniformity of the microtubular flagellar root system in various not closely related groups of green algae has been interpreted as reflecting a general important function of these systems and some functional explanations have been offered (Melkonian, 1980b).

Only few ultrastructural studies have been undertaken on motile cells of siphonalean green algae (Crawley, 1966; Burr \& West, 1970; Woodcock \& Miller, 1973; Moestrup \& Hoffman, 1975; Hori, 1977; Hori \& Enomoto, 1978; Roberts, 1980; Roberts et al., 1980; Roberts et al., 1981, in prep.) and these have not yet led to self-explanatory results. It has been noted that connecting fibres in Bryopsis are very similar to those found in Ulva and Enteromorpha (Melkonian, 1980a). From a study on the stephanokont zoospores and the anisogametes of Derbesia tenuissima, Roberts et al. (1980; 1981 in prep.) also concluded that the flagellar apparatus of the reproductive cells of Derbesia resembled that of ulvalean algae and therefore they tentatively included the siphonalean green algae in the Ulvaphyceae sensu Stewart \& Mattox. It is however clear that too few species of siphonalean green algae have been investigated in detail to make such a proposal sensible at this time.

The present study on the flagellar apparatus of female gametes of Bryopsis was partially undertaken to add to our knowledge about the flagellar apparatus in siphonalean green algae and partly because Bryopsis offers an excellent opportunity to study the effect of extreme anisogamy on the detailed structure of the flagellar apparatus. The male gametes have previously been studied in some detail (Hori, 1977; Melkonian, 1980a).

This study is the third contribution of a series (see also Melkonian, 1978, 1979), which aims to evaluate structural variability and functional implications of cruciate flagellar root systems in green algae.

\section{MATERIAL AND METHODS}

Cultures of female and male strains of Bryopsis lyngbyei Hornemann (Bryopsidales) were kindly provided by Dr. Dr. h. c. P. Kornmann (Helgoland). The algae were cultured in an Erd-Schreiber solution, containing $0.2 \mathrm{~g} \mathrm{NaNO}_{3}, 0.02 \mathrm{~g} \mathrm{Na}_{2} \mathrm{HPO}_{4} \times 10 \mathrm{H}_{2} \mathrm{O}$ and $50 \mathrm{ml}$ soil extract per 1 l of filtered seawater. The general culture condition were: $14 / 10 \mathrm{~h}$ light/dark cycle, $10^{\circ} \mathrm{C}$ and approximately 2500 Lux light intensity. Plastic petri dishes were used as culture vessels. Gamete discharge in the female gametangia usually occurs shortly after the beginning of the light period. After discharge, female gametes accumulated near the light source at the meniscus, where they could be collected as a concentrated suspension with a micropipette. The cells were fixed for electron microscopy as previously described (Melkonian, 1979). Fixed cells were washed with ErdSchreiber-solution $\left(4^{\circ} \mathrm{C}\right)$ and further processed by standard methods (Melkonian, 1975). Sections were cut with a diamond knife and observed in a Siemens Elmiskop IA. 


\section{RESULTS}

The biflagellate female gametes of Bryopsis lyngbyei measure around $9 \mu \mathrm{m}$ in length and $5 \mu \mathrm{m}$ in width and are pear-shaped. The general ultrastructure of the female gamete is very similar to that of female gametes of Bryopsis hypnoides (Burr \& West, 1970) and need not be described in detail. A longitudinal section of a female gamete

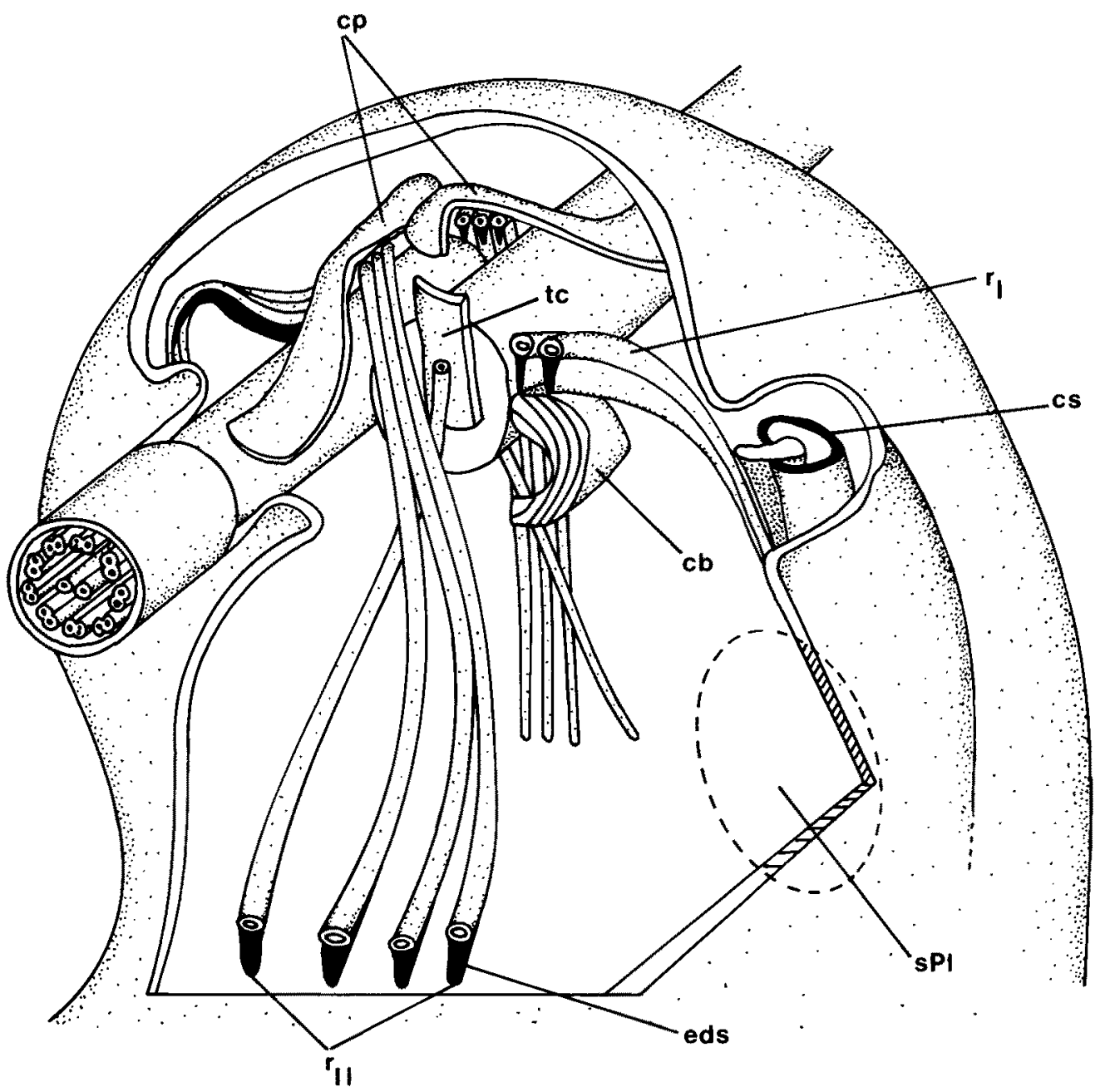

Fig. 1. Schematic 3-dimensional reconstruction of the flagellar apparatus of female gametes of Bryopsis lyngbyei. Abbreviations: $\mathrm{cp}=$ capping plate composed of two non-striated connecting fibres; tc $=$ terminal cap, partially closing the proximal end of each basal body; $\mathbf{r}_{1}=$ two-stranded microtubular root; $\mathrm{r}_{\mathrm{H}}=$ four-stranded microtubular root (sometimes with 5 microtubules); cs = cylindrical structure associated with the two-stranded microtubular root; $\mathrm{cb}=$ crescent body with striations; eds = electron dense sheets underlying each root microtubule of all four microtubular roots; sP1 = specialized area of the plasmalemma near the cylindrical structure (probably a mating structure) 
revealing distribution of major cell organelles might therefore be sufficient for the present purpose (Fig. 2).

A diagrammatic presentation of the flagellar apparatus is shown in Fig. 1 and may be used as a reference to the following description and subsequent micrographs.

Cross sections of the flagellar axoneme in the shaft region reveal in addition to the usual $9+2$ pattern of microtubules usually one bipartition in a B-tubule of one outer doublet per section (Fig. 8). The transition region of the flagellum is unusually long $(260-290 \mathrm{~nm})$ and in cross section exhibits the usual stellate pattern found in most green algae (Figs 6, 7). In longitudinal sections however the transitional region lacks the transverse diaphragm (Fig. 6). Each basal body is about $360-400 \mathrm{~nm}$ long and in cross sections a cartwheel pattern is observed in the proximal region of each basal body. The two basal bodies are parallel to one another (angle between them: $180^{\circ}$ ) and overlap at their proximal ends by a rather constant distance of $170 \mathrm{~nm}$ (Fig. 3).

Basal bodies are interconnected by two principal non-striated connecting fibres forming a capping plate (Fig. 4). Each connecting fibre arises from a different basal body but both touch each other midway between the basal bodies and thereby provide the connection between the two basal bodies. In most sections however these two fibres appear as a single capping plate (e. g. Fig. 4). The connecting fibres attach at the distal ends of the respective basal bodies and in cross sections through this area the fibre has a curved appearance and is linked to 4 different triplets of the basal body (Fig. 23). In addition each connecting fibre has one discrete attachment site to the plasmalemma at the most apical end of the cell (Figs 9, 17, 22). The compound capping plate therefore arches anteriorly to form a bridge with a horizontal mid-region between the two anchoring processes (e.g. Fig. 4). The lateral regions of the capping plate extend posteriolaterally. Whereas the length of the individual connecting fibres roughly corresponds to the overall length of the basal body (ca. $400 \mathrm{~nm}$ ) the width of the capping plate varies between 200 and $250 \mathrm{~nm}$. In the overlap region both basal bodies are linked together by electron dense material with inconspicuous striations (Fig. 3).

The proximal end of each basal body is partially closed by a terminal cap, i. e. an

Fig. 2. Longitudinal section through a female gamete revealing major organelle distribution. The chloroplast is posteriorly located, contains numerous starch grains, a pyrenoid and a very large eyespot (arrows). $\times 8500$

Fig. 3. Transverse section through the tip of the female gamete. $b=$ basal bodies; $2=$ two-stranded microtubular root; $3=$ three-stranded microtubular root; $4=$ four- (or five-)stranded microtubular roots; open arrow $=$ cylindrical structure in cross section associated with the two-stranded microtubular root. Notice basal body overlap. $\times 60000$

Fig. 4. Vertical longitudinal section through the flagellar apparatus. $b=$ basal body; the capping plate arches up towards the plasmalemma to which it links (arrow). $\times 52500$

Fig. 5. Oblique section through the flagellar apparatus. $m=$ mitochondrial profile; $3=$ threestranded microtubular root the four lines visible in this root type reflect oblique sections through the electron dense sheets that underly the root microtubules; these sheets may bifurcate [see e. $g$.

Fig. 18]; open arrow $=$ oblique section through the cylindrical structure. $\times 45000$

Fig. 6. Longitudinal section through transition region and basal body. The length of the transition region is indicated by two open arrows. $\times 67500$

Fig. 7. Cross section through the transition region revealing stellate pattern. $\times 82500$

Fig. 8. Cross section through the free part of a flagellum. Two opposite outer doublet exhibit microtubule septations in their B-tubules (arrows). $\times 82500$ 

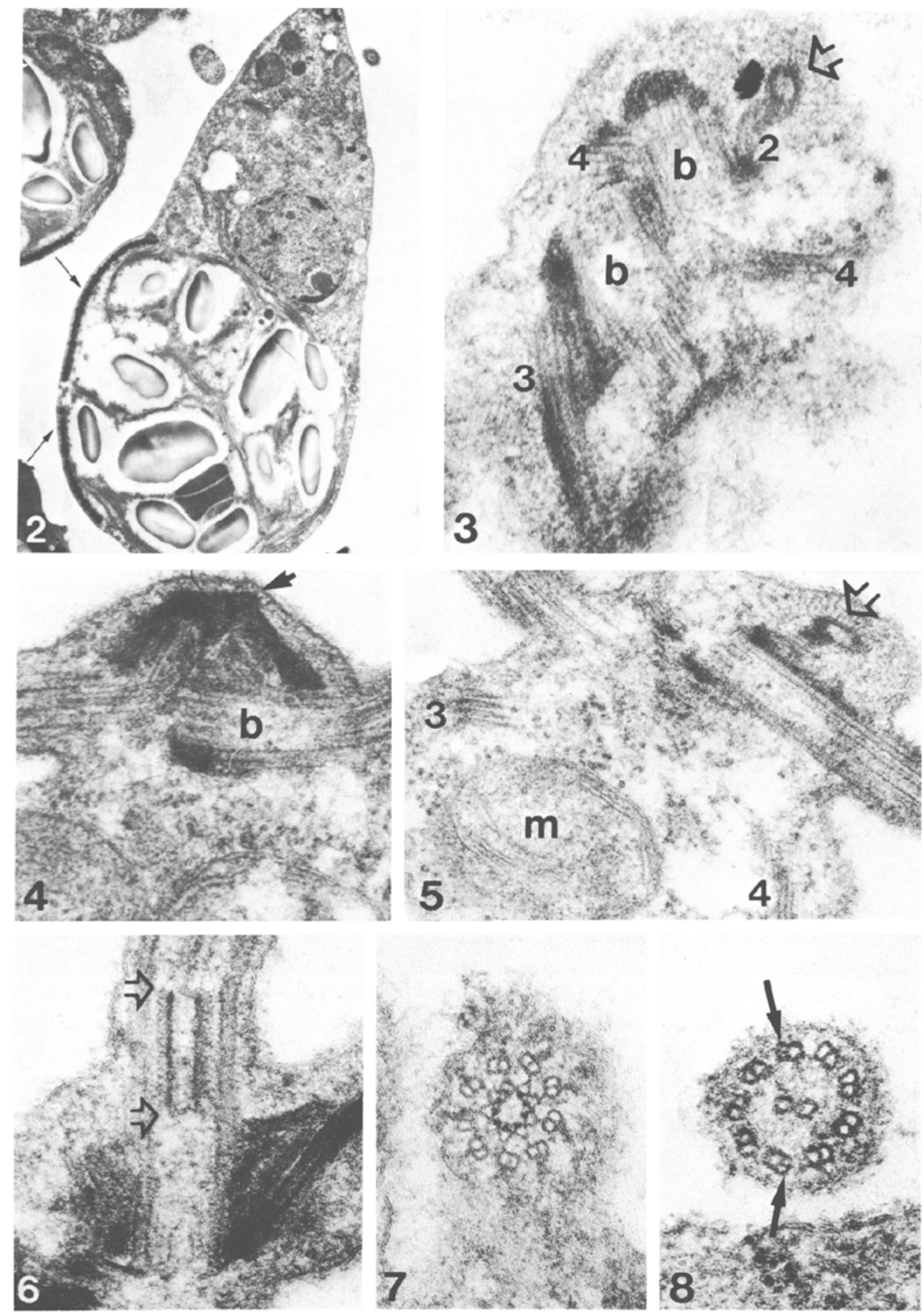
electron dense plate (Fig. 24). The terminal cap is $15-26 \mathrm{~nm}$ thick and is directly linked to the capping plate of the respective basal body.

Four microtubular roots make up the flagellar root system (Figs 3, 5). Each basal body is associated with two of the four roots. Transverse sections through the apical part of a female gamete show that the four roots are not arranged in a typical cruciate pattern, opposite roots are considerably displaced against each other and appear to take nonsymmetrical paths inside the cell (Figs 3,5). Furthermore at least two opposite roots are structurally not identical (Figs 3,5). One root is associated with an electron dense ring, three-dimensionally a cylinder (described in detail below), whereas the opposite root is not associated with such a structure and appears to contain more microtubules than the other root (Figs 3,5 ). The flagellar root system does not, therefore, show $180^{\circ}$ rotational symmetry.

The two structurally different opposite roots attach to the outer sides of the respective basal bodies (Figs 3,5,9,10, 22, 23).

Cross-sections through the root associated with the cylindrical structure (Figs 9-16) indicate that this root type consists of two microtubules along its length. The root microtubules are not directly connected to basal body triplets but through electron dense material (Figs 9, 11, 22, 23). In the proximal parts of the root microtubules, electron dense material over- and underlies the root microtubules (Figs 9-11). The underlying material forms a characteristic crescent body. In cross sections through the root the crescent body is 200-250 nm wide (Figs 9-11); the crescent shape, however, is only revealed when the root is sectioned in vertical, longitudinal direction (e. g. Fig. 17). The curvature of the crescent body is towards the cell interior (Fig. 17). Several striations in the crescent body are clearly discernible in such longitudinal sections (Fig. 17). A similar crescent body with slightly smaller dimensions is associated with the opposite root (Fig. 22). The twostranded root is linked to the crescent body by two electron dense sheets, which run the whole length of this microtubular root (Figs 9-16). One of these sheets often bifurcates (Figs 11, 13,15). The electron dense material above the two root microtubules attaches to the capping plate (Fig. 9) and also to the cylindrical structure (Figs 9-11).

The root opposite the two-stranded root in cross sections always consists of 3 microtubules (Figs 18-21). Identification of this root in cross sections is easy because it is

Figs 9-16. Cross sections through the two-stranded microtubular root and associated cylindrical structure from proximal (Fig. 9) to distal parts (Fig. 16). All micrographs $\times 75000$

Fig. 9. Cross section through two-stranded microtubular root near its origin. $\mathrm{b}=$ basal body; $\mathrm{cp}=$ capping plate; $\mathrm{cb}=$ electron dense crescent body; open arrow $=$ cylindrical structure in cross section associated with the two-stranded root by electron dense material

Fig. 10-12. Serial sections through the two-stranded microtubular root. The plasmalemma, overlying the cylindrical structure, exhibits a semicircular bulge (Fig. 12). In this area the crescent body terminates. The root tubules are intimately associated with the cylindrical structure

Figs 13, 14. In the posterior region of the cylindrical structure, an area of the plasmalemma, adjacent to the plasmalemma overlying the cylindrical structure, appears to be thickened (arrows in Figs $13,14)$

Figs 15, 16. Cross section through distal parts of the two-stranded microtubular root. The cylindrical structure has terminated. In Fig. 15 electron dense material is still associated with the root microtubules. Electron dense sheets underlying individual root microtubules often show bifurcations (arrows). In Fig. 16. The root microtubules are only accompanied by the electron dense sheets 

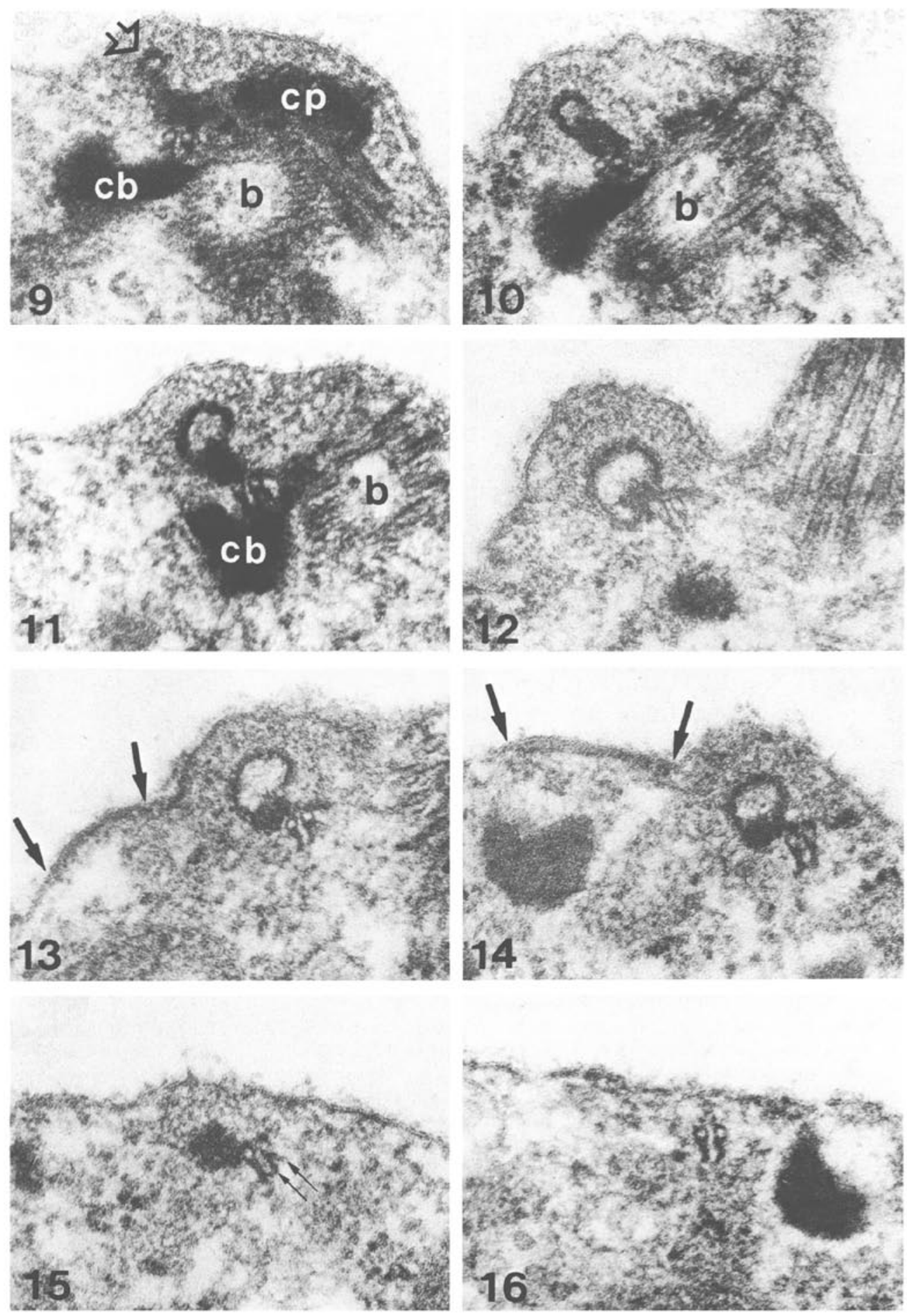
proximally associated with a crescent body (the other two roots lack a crescent body), but lacks the cylindrical structure (Fig 18-21). Overlying electron dense material connects this root to the basal body (Fig. 19). Each microtubule of the three-stranded root links to the crescent body through an electron dense sheet (Fig. 18). These sheets run the whole length of the three-stranded root and sometimes they also bifurcate (Figs 18-21).

The other two opposite microtubular roots approach the basal bodies in their overlap region approximately at right angles to the longitudinal plane of the two basal bodies (see also Fig. 1). They run very close to the proximal end of one basal body (Fig. 24) and appear to terminate at the connecting fibre of the other basal body (Fig. 4), in an area where both connecting fibres touch each other. In the most proximal cross sections, these roots exhibit typical root tubule configurations and a $3+1$ or $4+1$-pattern is present (Figs 24-26). The tubule of the second row (tubule 1, terminology according to Melkonian, 1978) is directly connected to the terminal cap of one basal body (Fig 24), the other three or four microtubules link to the capping plate (Fig. 24). In more distal cross sections through these roots, root tubule 1 changes position relative to the other microtubules and joins the upper row (Figs 25-27). In this root type, each microtubule is again associated with an electron dense sheet of similar structure as in the other two roots (Figs 24-27); overlying electron dense material is also present but only in the more distal parts of these roots (Figs 26, 27). A crescent body is absent and opposite roots appear to be identical in substructure. A few secondary cytoskeletal microtubules are sometimes seen to originate near the root microtubules (Fig. 25).

The cylindrical structure which is associated with the two-stranded microtubular root is the most conspicuous feature of the flagellar apparatus of the female gametes (Figs. 9-16, Fig. 17). Figures 9-14 represent cross sections through the cylindrical structure from its proximal (Fig. 9) to its distal (Fig. 14) end. In longitudinal section, the cylindrical structure measures around $1.4 \mu \mathrm{m}$ in length (Fig. 17). The diameter of the electron dense ring is variable, depending upon which part of the structure is sectioned. The central region has a maximum diameter of $160 \mathrm{~nm}$ (Fig. 12). The ring is not continuous, but in an area of $60 \mathrm{~nm}$ width, amorphous electron dense material penetrates into the ring interior (Figs 11-13). Electron dense material overlying the two root

Fig. 17. Longitudinal section through the cylindrical structure (limits of the cylindrical structure are given by the open arrows). $\mathrm{b}=$ basal bodies; $\mathrm{cb}=$ two crescent bodies of opposite roots; $\mathrm{cp}=$ capping plate; $m=$ mitochondrial profile; 2 = longitudinal section through two-stranded microtubular root. $\times 67500$

Figs 18-21. Cross sections through the three-stranded root (the root opposite the two-stranded root) from proximal (Fig. 18) to distal (Fig. 21) regions. All micrographs $\times 75000$

Fig. 18. Cross section through proximal parts of the three-stranded root. Electron dense sheets link individual root tubules to the underlying crescent body (cb). b= basal body; notice bifurcation of one of the electron dense sheets. Although there is some fuzzy material overlying the root microtubules (Figs 19,20) there is no cylindrical structure present in this root type.

Fig. 22. Cross section through the central region of the flagellar apparatus $b=$ basal bodies in their overlap region; $3=$ oblique section of three-stranded microtubular root; open arrow $=$ oblique section through cylindrical struture. Only one of the two opposite roots is associated with a cylindrical structure, but both are associated with a crescent body (cb) $\times 60000$

Fig. 23. Cross section through a basal body. Long arrow = capping plate in cross section apparently linked to 4 microtubule triplets of the basal body; open arrow = cylindrical structure in oblique section. $\times 75000$ 

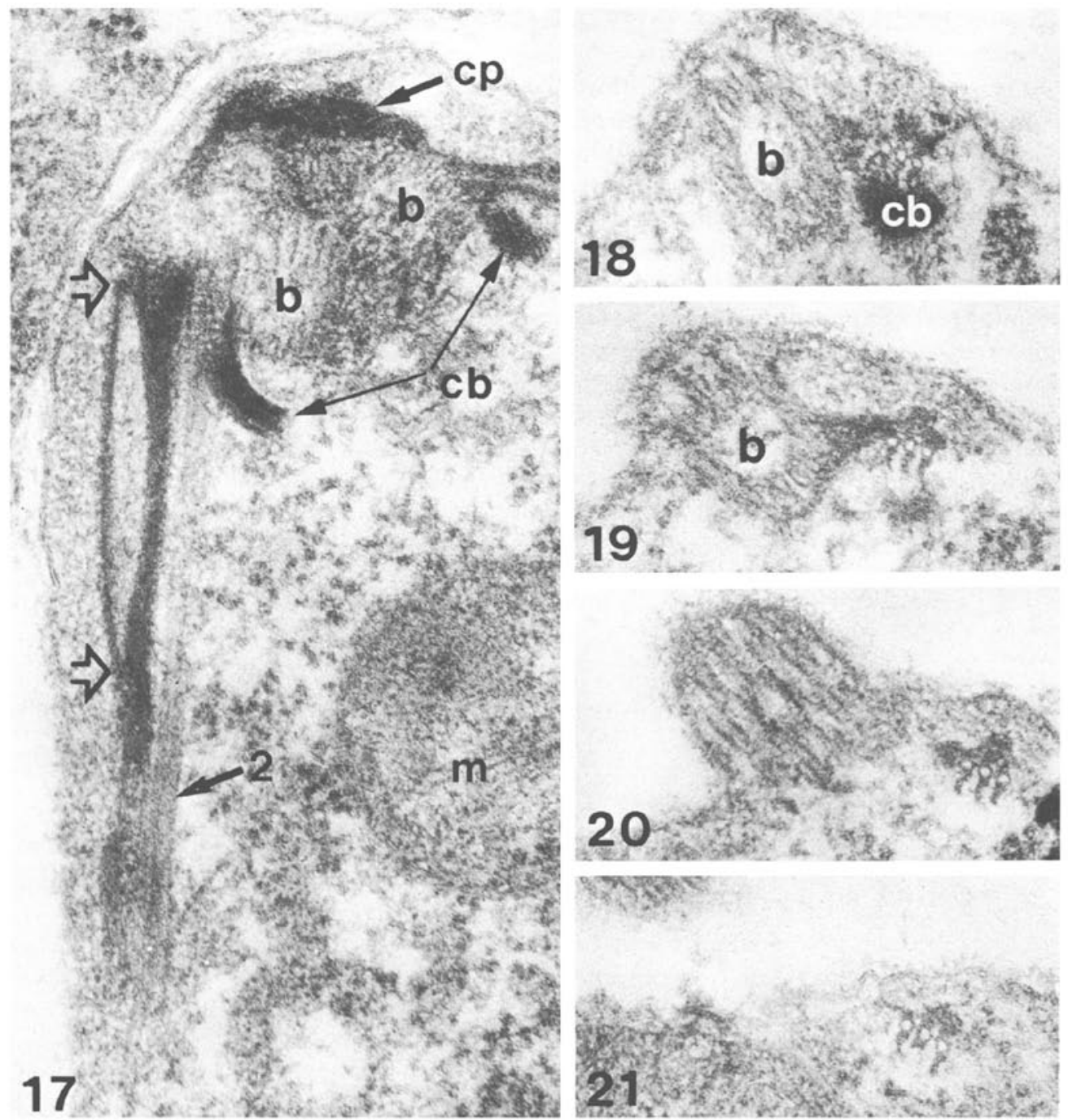

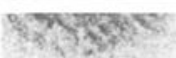
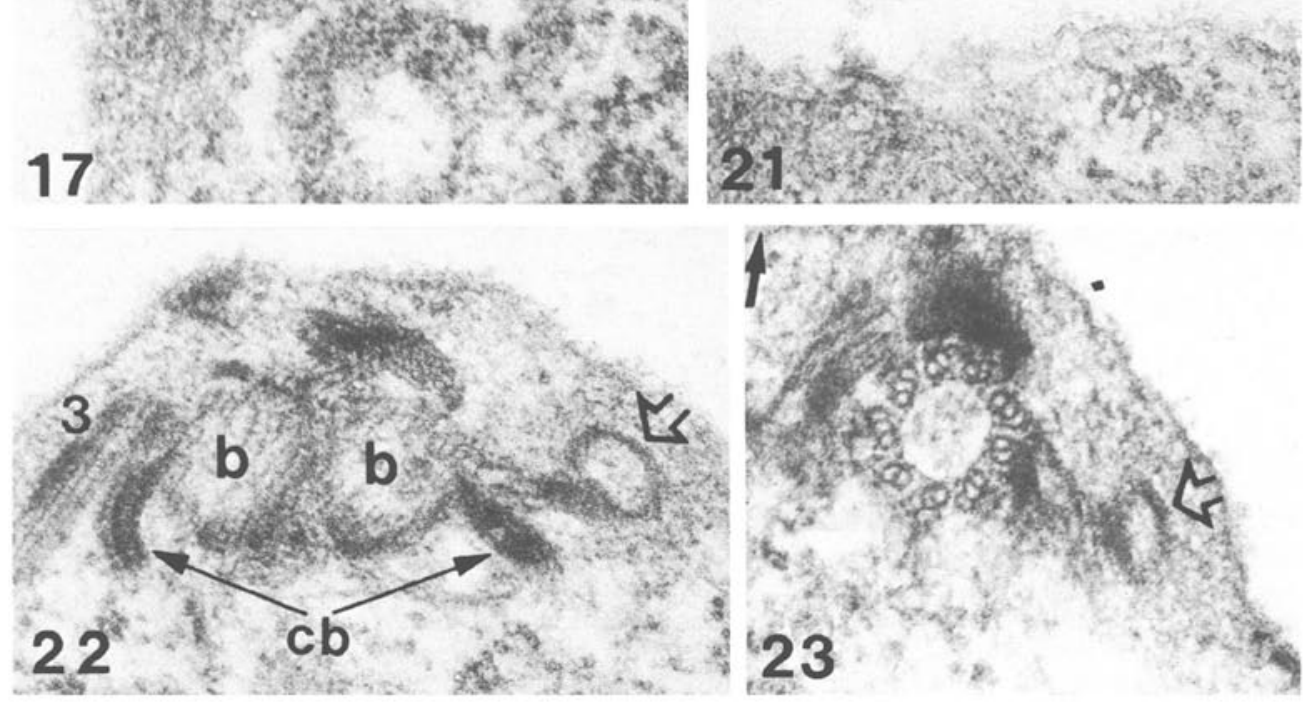
microtubules link these tubules to the amorphous material (Figs 11-14). The cylindrical structure causes the plasmalemma overlying the structure to bulge considerably outwards so that a semicylindrical protrusion of the cell surface is formed (Figs 12, 13). Towards the plasmalemma, the electron dense ring is surrounded by granular material (Figs 9-14). Adjacent to the semicylindrical plasmalemma area in some cross sections, part of the plasmalemma facing away from the flagellar apparatus appeared to be thickened (Figs 13, 14, arrows). This plasmalemma region in cross sections measures $0.3-0.4 \mu \mathrm{m}$. This area is confined to the posterior region of the semicircular bulge.

The electron dense material, overlying the root microtubules in some horizontal longitudinal sections, appeared to be striated, it was however not possible to determine the striation pattern nor to decide if 4 or only two striated roots are present. System II fibres (terminology according to Melkonian, 1980a) were not found in the flagellar apparatus of female gametes.
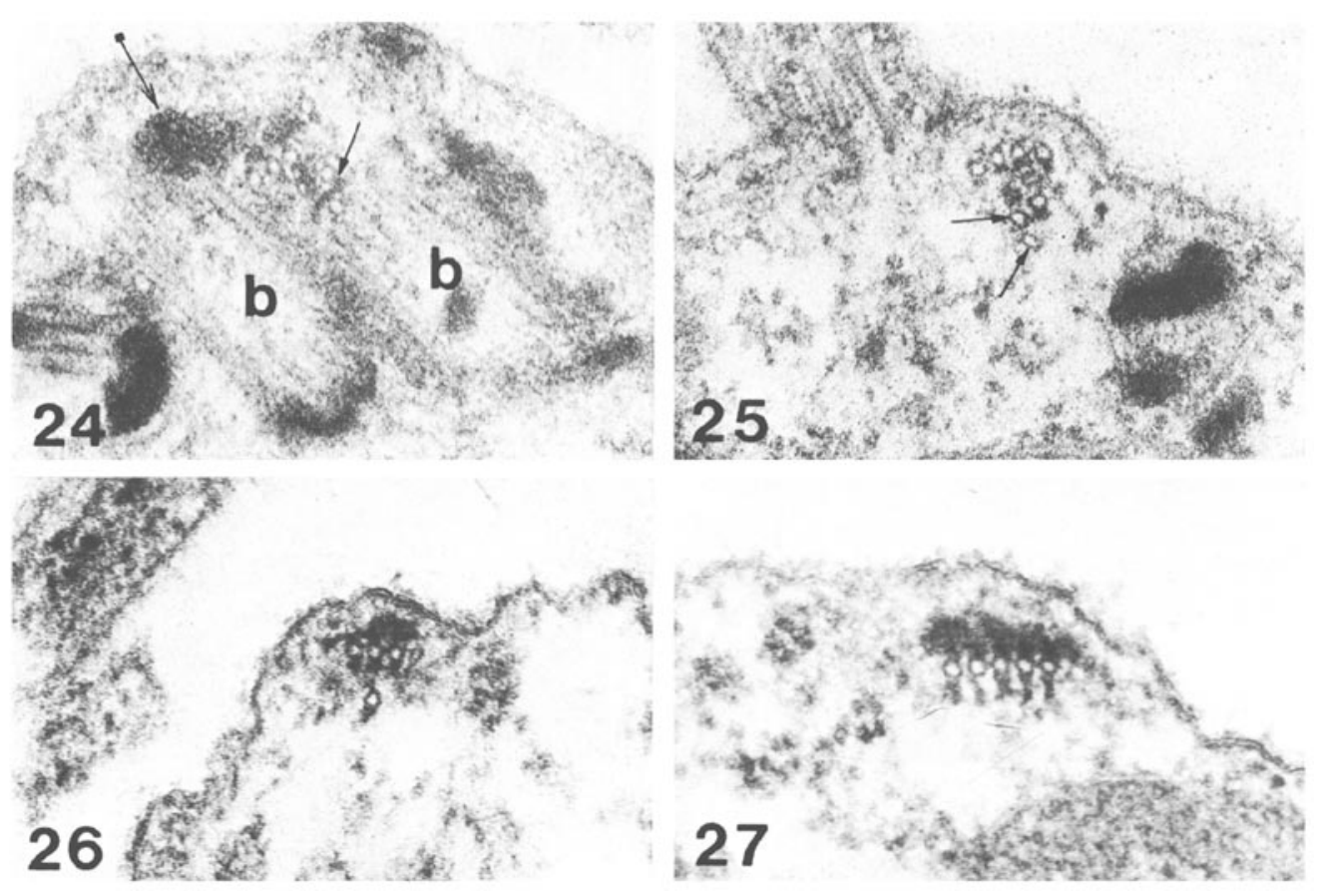

Figs 24-27. Cross section through root type II (four- or five-stranded root) from more proximal (Fig. 24) to more distal (Fig. 27) regions. All micrographs $\times 75000$

Fig. 24. Cross sections through 5-stranded root in the region of basal body overlap. $b=$ basal bodies; long arrow = capping plate; small arrow = root tubule 1 links to the terminal cap

Fig. 25. More distal cross section through 5-stranded root. Two secondary cytoplasmic microtubules (arrows) apparently originate at this root. In this region no electron dense material overlies the root microtubules

Figs 26,27 . Gradual change in root tubule configuration, root tubule 1 (root tubule in the second row) forms one row with the other four microtubules. Notice electron dense sheets associated with 


\section{DISCUSSION}

\section{Functional considerations}

The flagellar apparatus of female gametes of Bryopsis lyngbyei shows many unusual features that have not yet been found in other green algae. One of the more conspicuous structures in the flagellar apparatus is the cylindrical structure associated with the twostranded microtubular root. This structure is not present in the flagellar apparatus of male gametes in Bryopsis lyngbyei (Melkonian, 1980a and unpublished observations) or Bryopsis maxima (Hori, 1977). The structure is singular and connected to a specialized region of the plasmalemma near the flagellar bases. It is suggested that it represents a mating structure, an organelle associated with the plasmalemma of gametes and engaged in the gametic fusion process (Goodenough \& Weiss, 1975, Melkonian, 1980b). The mating structures of Chlamydomonas reinhardii (Goodenough \& Weiss, 1975) and Ulva lactuca (Melkonian, 1980b), which have been studied in detail, are also associated with a two-stranded microtubular root. Unfortunately, direct proof that the cylindrical structure acts as a mating structure during gametic fusion in Bryopsis is lacking, because gametic fusion in our cultures was a rare event and sufficient quantities of gamete pairs were not available. The absence of a defined mating structure in male gametes suggests that gametic fusion might occur in a way, which is different to the well known mechanisms of Chlamydomonas reinhardii (review by Goodenough, 1977) or Ulva lactuca (Melkonian, 1980b). In this context it is interesting to notice that in the oogamous gametic fusion of Prasiola stipitata (Friedmann \& Manton, 1960) the male gametes make contact with the eggs by one of their flagella. Male gametes of Bryopsis Iyngbyei exhibit an unusual swimming behaviour in which one of the two flagella is held in an anterior position, whereas the other flagellum is trailing (unpublished observations). It might well be that gametic fusion in Bryopsis is initiated in a similar way as in Prasiola.

The association of a two-stranded microtubular root with a mating structure and our unpublished observations that a 4- or 5-stranded microtubular root is often seen near the eyespot of the female gametes of Bryopsis lyngbyei, further add to the idea that microtubular flagellar roots might be essential for determining the exact position of both the mating structure and the eyespot in motile cells of many green algae (see also Melkonian, 1981).

An intriguing observation with respect to the cruciate microtubular root system in female gametes of Bryopsis lyngbyei is that a strict $180^{\circ}$ rotational symmetry (as defined by Floyd et al., 1980) is absent. It has previously been assumed that the microtubular roots in cruciate flagellar root systems, as well as the basal body-associated fibrous structures, exhibit $180^{\circ}$ rotational symmetry (Floyd et al., 1980). In green algae a $180^{\circ}$ rotational symmetry of the flagellar apparatus was not found in female gametes of Ulva lactuca, in which fibrous roots are unequally distributed on opposite sides of the flagellar apparatus (Melkonian, 1980b). The single eyespot and mating structure impose a typical left-right symmetry onto most green algal cells. In gametes of Ulva lactuca it has been shown that the eyespot and mating structure are located on the same side of the cell if one uses the plane of beat of the two flagella to separate a cell into two halves (Robenek \& Melkonian, 1981). The present observation, that one pair of opposite microtubular roots contains different microtubule numbers in the individual roots, appears to be the 
first report in the green algae. If one assumes that microtubule numbers in a root are functionally important (at least with respect to the two-stranded root only one exception has been noted, see Moestrup, 1978), an identical replication of this root becomes a necessity. Variation of root tubule numbers in opposite roots could then mean that either one or both of these roots are non-functional in the original sense. Evidence for such an assumption also comes from a recent observation that in zoospores of a species lacking an eyespot, root tubule numbers in the respective root type are more variable in opposite roots than in related species with zoospores exhibiting an eyespot (Melkonian, in preparation).

\section{Phylogenetic considerations}

Before the detailed structure of the flagellar apparatus in female gametes of Bryopsis lyngbyei is compared with that of other green algae, it is essential to compare it with the flagellar apparatus of the male gametes. Bryopsis gametes show an extreme case of anisogamy and the differences found in the flagellar apparatus between female and male gametes might be a reflection of this anisogamy. It has already been noted that male gametes lack the cylindrical structure, presumably a mating structure (see above). System II fibres are present in the male gametes of Bryopsis lyngbyei and Bryopsis maxima (Melkonian 1980a; Hori, 1977) associated with a microtubular root containing 4 microtubules (unpubl. observations). No system II fibres have been found in the flagellar apparatus of female gametes. They might be functional in the peculiar swimming behaviour of the male gametes (see above). On the other hand, the crescent body underlying two microtubular roots in the female gamete is not present in the male gamete. Differences in the detailed structure of the microtubular roots might exist, but no detailed information on microtubular roots in the male gametes exists. In summary both gamete types appear to have the following features of their flagellar apparatus in common: (a) a compound non-striated capping plate, (b) terminal caps, (c) microtubule septations in B-tubules of outer doublets, (d) an unusually long transition region with absence of a transverse diaphragm, (e) basal body overlap, (f) a cruciate microtubular root system in which the two-stranded roots link to the side of each basal body that is not adjacent to the other basal body.

Recently Roberts et al. (1981, in prep.) have comparatively studied the flagellar apparatus of the anisogametes of Derbesia tenuissima. The flagellar apparatus of the male gametes is nearly identical to that of Bryopsis male gametes. The female gametes of Derbesia tenuissima, however, have some peculiarities in the structure of their flagellar apparatus. The microtubular root system is cruciate with a 5-3-5-3-pattern and therefore differs from that described in this study. The path of the roots and the way in which they originate at the basal bodies suggests that the 5-stranded roots correspond to the 2- and 3-stranded roots of female gametes of Bryopsis lyngbyei, whereas the 3stranded roots correspond to the 4-(5-)stranded roots of male and female gametes of Bryopsis lyngbyei. An explanation for this heterogeneity of the microtubular root systems of female gametes between Bryopsis and Derbesia is difficult to give. It should however be noted that an eyespot is lacking in female gametes of Derbesia and the cylindrical structure also appears to be absent in female gametes of Derbesia. It is again suggestive that a microtubular root system that is not associated with an eyespot or mating structure would be modified during evolution. In addition, Roberts et al. (in 
preparation) found a system II fibre in the female gametes of Derbesia tenuissima, but orientation of the fibre and association with the 5-stranded root, seems to indicate that the fibre is not homologous to the system II fibre found in the male gametes of both genera and may be more related to the crescent body of female gametes of Bryopsis lyngbyei. Otherwise the structure of the flagellar apparatus of anisogametes of Derbesia tenuissima agrees well with the general characteristics given above (a)-(f) for the flagellar apparatus of anisogametes of Bryopsis.

If one compares the flagellar apparatus of Bryopsis gametes with that of other green algae it is obvious that the system is most similar to that of some green algae that have recently been classified as a separate class, the Ulvaphyceae sensu Stewart \& Mattox (Stewart \& Mattox, 1978; Sluiman et al., 1980). The features that the flagellar apparatus of Bryopsis gametes share with that of the Ulvaphyceae sensu Stewart \& Mattox include the capping plate, terminal caps, microtubule septations, basal body overlap, positional relation of root types to basal bodies and in part transition region ultrastructure. The Ulvaphyceae are additionally characterized by the presence of system I and system II fibrous roots (Melkonian, 1980a; SMAC or rhizoplasts in the terminology of Floyd et al., 1980). System I fibres have not been conclusively demonstrated in female gametes of Bryopsis, but the overlying electron dense material associated with all four roots might well be striated in horizontal longitudinal section. A system I fibre associated with a twostranded root was found in zoospores of Derbesia tenuissima (Roberts et al., 1980). System II fibres appear to be restricted to male gametes of siphonalean green algae (Hori, 1977; Melkonian 1980a; Roberts et al., 1981, in prep.). It is therefore not clear whether homology exists between the system II fibres of male gametes of siphonalean green algae and the prominent system II fibres of the Ulvaphyceae sensu Stewart \& Mattox.

The Chlorophyceae sensu Stewart \& Mattox (Stewart \& Mattox, 1975) have a differently constructed flagellar apparatus, including presence of distal and proximal striated connecting fibres, absence of terminal caps and microtubule septations and basal body overlap, lack of system II fibres (at least in the advanced members of this group) and a different ultrastructure of the transition region. The positional relation of root types to the respective basal bodies is also different in the Chlorophyceae sensu Stewart \& Mattox and since this character has previously not been used for phylogenetic considerations, some remarks should be made. In the Ulvaphyceae sensu Stewart \& Mattox, in Bryopsis, Derbesia and some other green algae related to each other (Microthamnion, Friedmannia, Trebouxia, Pleurastrum, the Chroolepidaceae; Melkonian, in preparation) basal body overlap results in the formation of two different sides of each basal body in a basal body pair: an outer side, facing away from the other basal body, and an inner side to which the other basal body in a proximal region attaches. It is found that in these groups of green algae two-stranded microtubular roots approach and link to the outer side of each basal body, whereas multi-membered roots (e. g. 4- or 5-stranded roots) approach a region between both basal bodies and link to the inner side of each basal body. In the Chlorophyceae sensu Stewart \& Mattox, the opposite situation occurs. Although there is no basal body overlap in the Chlorophyceae, the two basal bodies of a pair are displaced against each other by a distance of a basal body diameter or slightly less. Two-stranded microtubular roots approach the area between the basal bodies, opposite roots forming a straight line (e. g. Melkonian, 1978). Multi-membered roots 
approach each basal body at an angle of around $45^{\circ}$; opposite roots are considerably displaced against each other (Melkonian, 1978).

In summary, this study has shown that the flagellar apparatus of female gametes of Bryopsis lyngbyei is unique and contains structures not previously seen in other green algae, but in some general aspects resembles the flagellar apparatus of the Ulvaphyceae sensu Stewart \& Mattox. More siphonalen green algae should be studied with respect to the detailed structure of the flagellar apparatus in biflagellate cells, before final conclusions about the phylogenetic affinities of this green algal group can be made.

Acknowledgements. I would especially like to thank Dr. Dr, h. c. P. Kornmann (Helgoland) for donating the cultures of Bryopsis lyngbyei and his continuous interest in this study. Mrs. I. Wachholz, Mrs. E. Manshard and Mrs. B. Surek have helped during various stages of the work.

\section{LITERATURE CITED}

Burr, F. A. \& West, J. A., 1970. Light and electron microscope observations on the vegetative and reproductive structure of Bryopsis hypnoides. - Phycologia 9, 17-37.

Crawley, J. C. W., 1966. Some observations on the fine structure of the gametes and zygotes of Acetabularia. - Planta 69,365-376.

Floyd, G. L., Hoops, H. J. \& Swanson, J. A, 1980. Fine structure of the zoospore of Ulothrix belkae with emphasis on the flagellar apparatus. - Protoplasma 104, 17-32.

Friedmann, I. \& Manton, I., 1960. Gametes, fertilization and zygote development in Prasiola stipitata Suhr. - Nova Hedwigia 1, 1-32.

Goodenough, U. W., 1977. Mating interactions in Chlamydomonas. In: Microbial interactions. Ed. by J. L. Reissig. Chapman \& Hall, London, 323-350.

Goodenough, U. W. \& Weiss, R. L., 1975. Gametic differentiation in Chlamydomonas reinhardii. III: Cell wall lysis and microfilament-associated mating structure activation in wild-type and mutant strains, - J. Cell Biol. 67, 623-637.

Hoops, H. J., Floyd, G. L. \& Swanson, J. A., 1981. The ultrastructure of the biflagellate motile cells of Ulvaria oxysperma (Kutz.) Bliding and phylogenetic relationships among Ulvaphycean algae. Am. J. Bot. (In press).

Hori, T., 1977. Electron microscope observations on the flagellar apparatus of Bryopsis maxima (Chlorophyceae). - J. Phycol. 13, 238-243.

Hori, T. \& Enomoto, S., 1978. Developmental cytology of Dictyosphaeria cavernosa. I. Light and electron microscope observations on cytoplasmic cleavage in zooid formation. - Botanica mar. $21,401-408$.

Melkonian, M., 1975. The fine structure of the zoospores of Fritschiella tuberosa Iyeng. (Chaetophorineae, Chlorophyceae) with special reference to the flagellar apparatus. - Protoplasma $86,391-404$.

Melkonian, M., 1978. Structure and significance of cruciate flagellar root systems in green algae: comparative investigations in species of Chlorosarcinopsis (Chlorosarcinales). - Pl. Syst. Evol. $130,265-292$.

Melkonian, M., 1979. Structure and significance of cruciate flagellar root systems in green algae: zoospores of Ulva lactuca (Ulvales, Chlorophyceae). - Helgoländer wiss. Meeresunters. 32, $425-435$,

Melkonian, M., 1980a. Ultrastructural aspects of basal body associated fibrous structures in green algae: a critical review. - BioSystems 12, 85-104.

Melkonian, M., 1980b. Flagellar roots, mating structure and gametic fusion in the green alga Ulva lactuca (Ulvales). - J. Cell Sci. 46, 149-169.

Melkonian, M., 1981. The functional analysis of the flagellar apparatus in green algae. In: Eucaryotic and procaryotic flagella. Ed. by Amos, W. B. \& Duckett, J. G., Cambridge Univ. Press, Cambridge (in press). 
Moestrup, $\varnothing ., 1978$. On the phylogenetic validity of the flagellar apparatus in green algae and other chlorophyll $\mathrm{a}$ and $\mathrm{b}$ containing plants. - BioSystems $10,117-144$.

Moestrup, $\varnothing$. \& Hofman, L. R., 1975. A study of the spermatozoids of Dichotomosiphon tuberosus (Chlorophyceae). - J. Phycol. 11, 225-235.

Pickett-Heaps, J. D., 1975. Green algae. Sinauer, Sunderland, Mass., 606 pp.

Robenek, H. \& Melkonian, M. 1981. Comparative ultrastructure of eyespot membranes in gametes and zoospores of the green alga Ulva lactuca (Ulvales). - J. Cell Sci. (In press).

Roberts, K. R, 1980. Ultrastructure of the flagellated cells of Derbesia tenuissima. - J. Phycol. 16 (Suppl.), 132.

Roberts, K. R., Sluiman, H. J., Stewart, K. D. \& Mattox, K. R., 1980. Comparative cytology and taxonomy of the Ulvaphyceae. II. Ulvalean characteristics of the stephanokont flagellar apparatus of Derbesia tenuissima. - Protoplasma 104, 223-238.

Sluiman, H. J., Roberts, K. R., Stewart, K. D. \& Mattox, K. R., 1980. Comparative cytology and taxonomy of Ulothrix. I. The zoospore of $U$. zonata. - J. Phycol. (In press).

Stewart, K. D. \& Mattox, K. R, 1975. Comparative cytology, evolution and classification of the green algae with some consideration of the origin of other organisms with chlorophyll a and b. - Bot. Rev. 41, 104-135.

Stewart, K. D. \& Mattox, K. R., 1978. Structural evolution in the flagellated cells of green algae and land plants. - BioSystems 10, 145-152.

Woodcock, C. L. F. \& Miller, G. J., 1973. Ultrastructural features of the life cycle of Acetabularia mediterranea. I. Gametogenesis. - Protoplasma 77, 313-329. 